\title{
What is the Relationship between Health, Mood, and Mild Cognitive Impairment?
}

\author{
Jennifer A. Yates ${ }^{\mathrm{a}, *}$, Linda Clare ${ }^{\mathrm{b}}$ and Robert T. Woods ${ }^{\mathrm{c}}$ in collaboration with The Cognitive \\ Function and Ageing Study: Wales \\ ${ }^{\mathrm{a}}$ Institute of Mental Health, University of Nottingham, Nottingham, UK \\ ${ }^{\mathrm{b}}$ Centre for Research in Ageing and Cognitive Health, School of Psychology, and PenCLAHRC, \\ University of Exeter Medical School, Exeter, UK \\ ${ }^{\mathrm{c}}$ Dementia Services Development Centre, Bangor University, Bangor, UK
}

Accepted 19 September 2016

\begin{abstract}
Mild cognitive impairment (MCI) often co-exists with mood problems, and both cognitive functioning and mood are known to be linked with health. This study aims to investigate how health, mood, and cognitive impairment interact. Health is often assessed using a single proxy measure, but the use of a range of measures can provide a more informative picture and allows for combination into a comprehensive measure of health. We report an analysis of data from the Cognitive Function and Ageing Study Wales (CFAS Wales, N=3,173), in which structured interviews with older people captured measures of cognition, mood, and health. Each measure of health was assessed independently in relation to cognition and mood, and then all measures were combined to form a latent health variable and tested using structural equation modeling (SEM). SEM confirmed the association between health and cognition, with depression acting as a mediator. All measures of health were individually associated with levels of anxiety and depression. Participants reporting mood problems were less likely to engage in physical activity and more likely to report poor or fair health, have more comorbid health conditions, use more services, and experience difficulties with instrumental activities of daily living. Perceived health was associated with cognitive status; participants with MCI were more likely to report fair or poor health than participants who were cognitively unimpaired. Careful intervention and encouragement to maintain healthy lifestyles as people age could help to reduce the risk of both mood problems and cognitive decline.
\end{abstract}

Keywords: Anxiety, cognitive impairment, depression, health

\section{INTRODUCTION}

Mild cognitive impairment (MCI), a transitional state between normal and pathological aging [1], has been linked with both mood and health. Evidence suggests that MCI is related to depression and anxiety [2-5], that mood and health are related [6-10], and that cognitive functioning and health are related [11-14] in older people. However, no studies have investigated the three-way associations between MCI, mood, and health.

\footnotetext{
*Correspondence to: Dr. Jennifer A. Yates, D20 Institute of Mental Health, Triumph Road, Jubilee Campus, University of Nottingham, NG7 2TU, UK. Tel.: +44 011574 84255; Fax: +44 011582 31289; E-mail: Jennifer.yates@nottingham.ac.uk.
}

Health as a concept can be difficult to measure and consequently proxy measures such as physical activity [15], health service use [16], and ability to carry out instrumental activities of daily living (IADLS) are often used in health research. Many studies use one single measure of health to examine its relationship with either cognitive functioning or mood, but in order to address the difficulties involved in measuring health, and what health might mean to different people, it is important to consider a range of measures to ensure that a holistic picture of health is captured. Therefore, we have used a range of proxy measures-physical activity, health service use, ability to carry out IADLs, perceived health, and current health conditions-both individually and in combination in the form of a latent variable. 
Previous research by our team highlighted differences in participants who meet criteria for MCI and report subjective memory complaints (SMC), and those who otherwise meet criteria for MCI but do not report SMC $[17,18]$. It is possible that the presence or absence of SMC is related to an underlying metacognitive process that could also influence appraisals in other areas of functioning, including health. Therefore, the present study will also examine differences across the measures of health in participants with MCI who report SMC and those who do not report SMC, to explore whether such processes extend beyond appraisals of cognitive functioning and are instead part of a global appraisal of health. Research into the role of SMC and their relevance in the MCI diagnosis is growing [19], but as yet no study has considered the effects of subjective appraisal in other domains.

In order to investigate this gap in the literature surrounding MCI, mood, and health, this study adopted the following aims:

1. To explore associations between MCI, mood, and health using multiple measures of health to provide a comprehensive overview of how MCI and mood are related to different measures of health.

2. To construct a combined measure of health in the form of one latent variable and test it in a mediation model using structural equation modeling (SEM) to examine associations between MCI and health.

3. To examine differences in relation to self-reported mood and health between participants with MCI who report SMC and those who do not.

\section{METHODS}

\section{Design}

Mood, cognitive functioning, and health were examined using cross-sectional data from a large sample of community dwelling older people who participated in the Cognitive Functioning and Ageing Study Wales (CFAS Wales). CFAS Wales is a longitudinal population-based study which has gathered information about participants drawn from two research centers in urban and rural areas of Wales, investigating changes that people may experience as they age. Participants took part in face-to-face, structured interviews covering cognitive functioning, mood, health, social networks, and quality of life, which were usually conducted in their own homes by trained interviewers through the medium of English or Welsh, depending on the participant's preference. Additional details may be found on the CFAS Wales website (http://www.cfas.ac.uk/cfas-wales/). This study presents analyses from the first wave of data collection. Ethical approval was granted by the relevant NHS Ethics committees.

\section{Participants}

All individuals aged over 65 years living in the Gwynedd, Ynys Môn, and Neath Port Talbot areas of Wales were randomly sampled between 2011 and 2013 from general practitioner (GP) lists, yielding a total sample of 3,593 people. Participants were excluded from taking part if their GP felt that it would be inappropriate, for example if they were terminally ill, or if they were unable to provide informed consent. When participants were not able to engage in an interview, an informant was sought and asked if they would be able to provide information on the participant's behalf.

This study focuses on participants who are not cognitively impaired and those that might be categorized as having MCI (with or without SMC). Therefore, participants were excluded from these analyses if they had a diagnosis of dementia $(n=129)$, impaired ADLs $(n=52)$, or cognitive decline greater than that expected for a classification of MCI, but not meeting the criteria for dementia for other reasons, such as having intact ADLs (other cognitive impairment no dementia; OCIND; $n=152$, see Fig. 1). Nine participants were also excluded from analyses on the basis of incomplete data, resulting in 3,173 participants included in this analysis.

\section{Classification of cognitive status}

Cognitive status categories were defined using the cognitive status algorithm (Fig. 1). Participants classified as having MCI displayed an objective cognitive impairment, an absence of dementia, intact ADLs, intact general cognition (demonstrated by a score equal to or greater than 22 on the Mini-Mental State Examination [20]) and the presence of SMC, indicated by a positive response by the participant to a self-report question regarding memory difficulties and forgetting [45]. The MCIW category included all participants who would otherwise meet criteria for MCI, except that they did not report SMC. Objective cognitive impairment was defined using the Cambridge Cognition Examination [21], which formed 


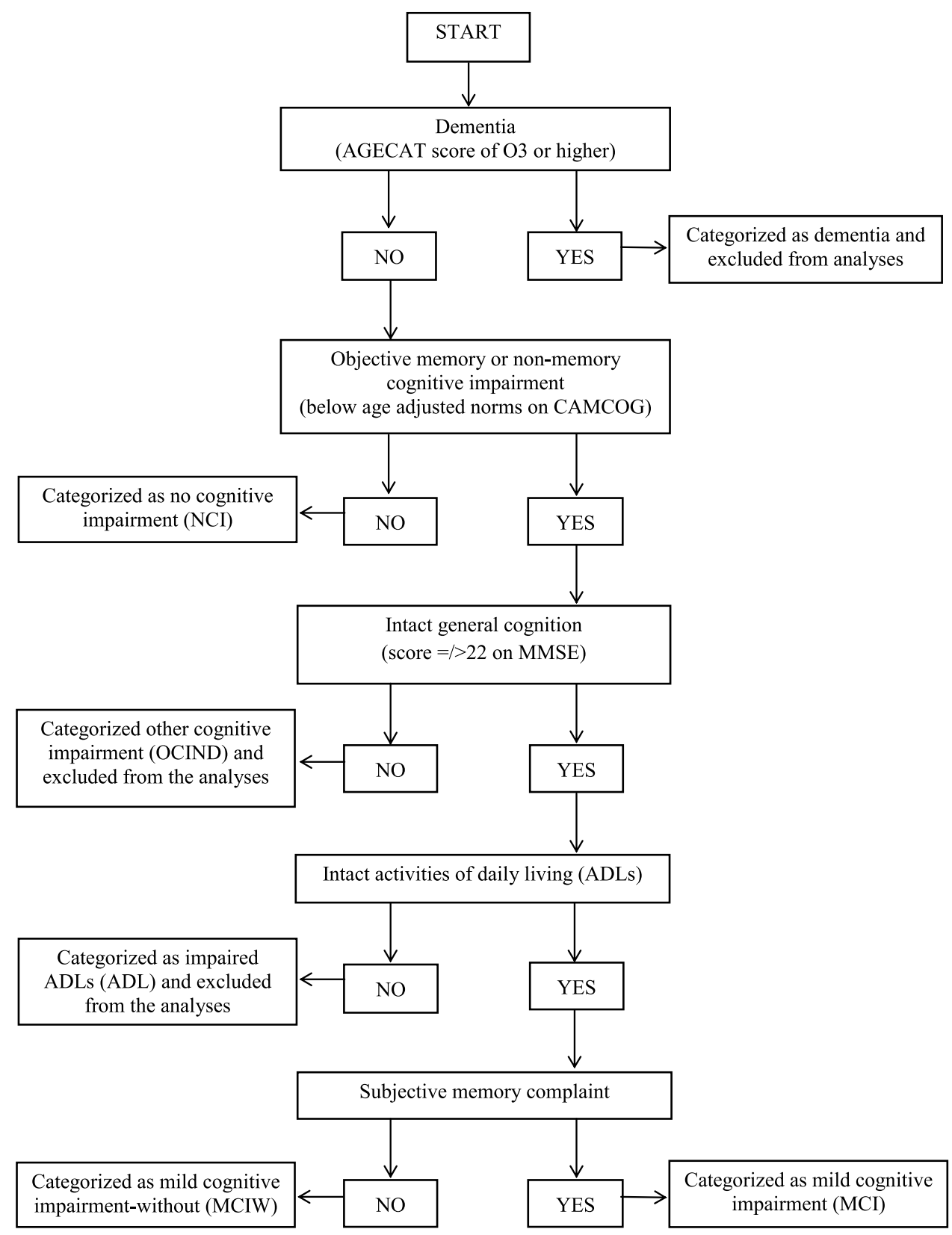

Fig. 1. Flow diagram of cognitive status algorithm.

a section of the CFAS Wales interview, with scores falling one standard deviation below age-adjusted norms on any of the cognitive domains taken to represent cognitive impairment.

\section{Assessment of health}

Five measures of health and health-related behavior were used to assess participants' overall health.
The five measures were based on questions asked during the CFAS Wales interview.

\section{Perceived health}

Subjectively-rated health was assessed with the question: "Would you say that for someone of your age, your own health in general is: Excellent/good/fair/poor/don't know?" 


\section{Number of health conditions}

Participants were asked whether or not they had the following health conditions: angina, intermittent claudication, high/low blood pressure, cancer, diabetes, Parkinson's disease, stroke, heart attack, fits/epilepsy, serious head injury, chronic bronchitis, asthma (excluding childhood asthma), arthritis, peptic ulcers, pernicious anemia, transient ischemic attack, thyroid problems, meningitis, and shingles. These questions were mapped onto the Charlson Comorbidity Index [22]. In line with the scoring system of the Charlson Co-Morbidity Index, a summary score was created by scoring each positive answer as one, except for cancer which scored two or three depending on whether the cancer was a past or current problem. The total score had a range from 0 to 21 , with higher scores reflecting higher levels of illness.

\section{Physical activity}

The physical activity score was calculated by asking how frequently participants took part in various activities with a total score that ranged from 0 to 72 .

\section{Service use}

Health service usage was investigated by asking participants if they had used certain services and how frequently. Positive answers were summed and adjusted for frequency, with higher scores indicating increased usage of services.

\section{Instrumental activities of daily living}

IADLs were assessed using five questions in the CFAS Wales interview: "Are you able to do the heavy housework?", "Are you able to shop and carry heavy bags?", "Do you have any difficulty managing money (paying bills/writing cheques or using an ATM to remove or deposit money)?", "Do you have any difficulty following TV programmes or movies and remembering details of the stories?", and "Have you needed any help recently to check your change after spending small amounts of money?". Participants responded by reporting whether they needed help, had some difficulty or did not have any difficulty with each activity. The total IADL score used in this analysis ranged from 0 to 10 and higher scores reflected less functionality.

\section{Assessment of mood}

Anxiety and depression were defined using the Geriatric Mental State Automated Geriatric Examination for Computer Assisted Taxonomy
(GMS-AGECAT) algorithm [23], where a score of two indicated mild symptoms and a score of three or above indicated a case of anxiety or depression. In this study, participants with a score of two or above were considered to have anxiety or depression.

\section{Statistical analyses}

Descriptive analyses were conducted using SPSS 20.0. Analysis of variance with planned contrasts and post hoc procedures and chi squared analyses were used to investigate the associations between cognitive status, mood and health, and logistic regression analyses were used to investigate changes in odds of experiencing mood problems according to health and cognitive status. The measures of health were combined and investigated as a latent variable in a mediation model using SEM procedures in Mplus Version 7. Sensitivity analyses and model testing were conducted to devise the best fitting model.

\section{RESULTS}

Participant characteristics are shown in Table 1. The participants were categorized as having no cognitive impairment (NCI), MCI, or MCIW. There were no differences between the cognitive groups in age, gender or years in full time education. Differences between the cognitive status categories on each measure of health are shown in Table 1. Differences between participants with and without anxiety or depression are shown in Table 2.

\section{Perceived health}

Pearson's chi square and likelihood ratios showed that there was a significant difference in perceived health between participants classified as NCI, MCI, and MCIW, $\left(\chi^{2}(6)=28.80, p<0.001\right)$. Logistic regression was used to investigate the association further using perceived health collapsed into two categories of excellent/good and fair/poor to allow for the calculation of risk for each cognitive status. The odds of reporting fair/poor health were significantly decreased for participants categorized as NCI $(\mathrm{OR}=0.71, \mathrm{CI}=0.58-0.87, p=0.001)$, but significantly increased for participants in the MCI category $(\mathrm{OR}=1.89, \mathrm{CI}=1.41-2.54, p<0.001)$. The odds were not significantly changed for participants in the MCIW category $(\mathrm{OR}=1.09, \mathrm{CI}=0.84-1.40$, $p=0.523$ ). Perceived health was found to be related 
Table 1

Descriptive statistics separated by cognitive status group

\begin{tabular}{|c|c|c|c|c|}
\hline & NCI & MCI & MCIW & F value ( $p$ value) \\
\hline Age, mean (SD) & $74.31(6.95)$ & $73.88(6.12)$ & $74.87(6.50)$ & \\
\hline Female, N (\%) & 1444 (54.6) & $89(44.5)$ & $196(59.6)$ & \\
\hline Years in full time education, mean (SD) & $11.83(2.80)$ & $11.44(2.40)$ & $11.08(2.31)$ & \\
\hline \multicolumn{5}{|l|}{ Perceived health N (\%) } \\
\hline Excellent & $590(22.3)$ & $30(15.0)$ & $75(22.8)$ & \\
\hline Good & $1255(47.5)$ & $78(39.0)$ & $134(40.7)$ & \\
\hline Fair & $607(23.0)$ & $60(30.0)$ & $82(24.9)$ & \\
\hline Poor & $159(6.0)$ & $26(13.0)$ & $17(5.2)$ & \\
\hline Physical activity, mean (SD) & $16.50(10.37)$ & $12.60(10.09)$ & $13.96(10.37)$ & $20.34(p<0.001)$ \\
\hline Service use, mean (SD) & $4.09(7.39)$ & $4.26(8.91)$ & $3.88(6.99)$ & $9.80(p=0.838)$ \\
\hline Health conditions, mean (SD) & $2.95(2.01)$ & $3.32(2.27)$ & $2.60(1.89)$ & $8.25(p<0.001)$ \\
\hline IADL score, $(\mathrm{SD})$ & $1.39(1.74)$ & $2.08(2.09)$ & $1.50(1.96)$ & $10.25(p<0.001)$ \\
\hline Anxiety, N (\%) & $133(5.03)$ & $18(9.0)$ & $12(3.6)$ & \\
\hline Depression, N (\%) & $628(23.8)$ & $78(39.0)$ & $82(24.9)$ & \\
\hline Subjective memory complaint, $\mathrm{N}(\%)$ & $850(32.1)$ & $200(100)$ & $0(0.0)$ & \\
\hline Total & 2644 & 200 & 329 & \\
\hline
\end{tabular}

NCI, no cognitive impairment; MCI, objective cognitive impairment, intact general cognition, intact activities of daily living, subjective memory complaint reported by the participant, absence of dementia; MCIW, objective cognitive impairment, intact general cognition, intact activities of daily living, absence of subjective memory complaint, absence of dementia; IADL, instrumental activities of daily living.

Table 2

Mean scores for measures of health for participants with and without anxiety or depression

\begin{tabular}{lccccrr}
\hline & No anxiety & Anxiety & T value $(p$ value $)$ & No depression & Depression & T value $(p$ value $)$ \\
\hline Health conditions mean (SD) & $2.87(1.99)$ & $4.11(2.24)$ & $-6.90(p<0.001)$ & $2.75(1.94)$ & $3.49(2.16)$ & $-8.47(p<0.001)$ \\
Physical activity mean (SD) & $16.21(10.39)$ & $12.01(10.08)$ & $5.04(p<0.001)$ & $16.80(10.36)$ & $13.53(10.18)$ & $7.72(p<0.001)$ \\
Service use mean (SD) & $3.96(7.44)$ & $6.18(7.44)$ & $-3.71(p<0.001)$ & $3.88(7.56)$ & $4.66(7.09)$ & $-2.62(p<0.001)$ \\
IADL score (SD) & $1.38(1.76)$ & $2.67(2.06)$ & $-7.84(p<0.001)$ & $1.23(1.68)$ & $2.09(1.98)$ & $-10.88(p<0.001)$ \\
\hline
\end{tabular}

IADL, instrumental activities of daily living.

to anxiety $\left(\chi^{2}(3)=126.37, p<0.001\right)$ and depression $\left(\chi^{2}(3)=151.73, p<0.001\right)$. Perceived health was again collapsed into two categories to calculate risk, and the association between anxiety and perceived health remained $\left(\chi^{2}(1)=85.86, p<0.001\right)$. Based on the odds ratio, the odds of having anxiety were 4.31 times higher when participants reported fair/poor health $(\mathrm{CI}=3.09-6.01, p<0.001)$. The association between depression and perceived health also remained $\left(\chi^{2}(1)=114.04, p<0.001\right)$ when perceived health was collapsed into two categories, with the odds of having depression 2.48 times higher for participants who reported fair/poor health $(\mathrm{CI}=2.10-2.94, p<0.001)$.

\section{Number of health conditions}

A significant effect of cognitive status was found on health conditions, $F(2,384.74)=8.25, p<0.001$ (equal variances not assumed), where participants with MCI reported the most health conditions, followed by those categorized as NCI, with those in the
MCIW category reporting the least. Planned contrasts revealed a significant difference in number of health conditions between participants with $\mathrm{MCI}$ and those categorized as MCIW, $t(363.30)=-3.81, p<0.001$ (equal variances not assumed) and this was confirmed by post hoc testing using the Games-Howell procedure. Planned contrasts failed to show significant differences between participants with cognitive impairment (MCI and MCIW categories combined), and those categorized as NCI, $t(492.23)=0.10$, $p=0.918$ (equal variances not assumed). However, further post hoc testing with the Games-Howell procedure revealed a significant mean difference between the MCIW category and participants categorized as NCI. Participants with anxiety were significantly more likely to report health conditions than those without anxiety, $t(176.07)=-6.90$, $p<0.001, r=0.46$ (equal variances not assumed). Participants with symptoms of depression were also significantly more likely to report health conditions than those without depression, $t(1234.31)=-8.47$, $p<0.001, r=0.23$ (equal variances not assumed). 


\section{Physical activity}

A one-way ANOVA showed that there was a significant effect of cognitive status on levels of physical activity, $F(2,3172)=20.34, p<0.001$. Planned contrasts revealed that having cognitive impairment (either MCI or MCIW) was associated with decreased physical activity score compared to people with NCI, $t(3170)=-6.38, p<0.001, r=0.11$. However, there was no significant difference in physical activity score between people with MCI and MCIW, $t(3170)=1.47, p=0.142$. Post hoc tests confirmed that the means were significantly different between $\mathrm{NCI}$ and MCI, and NCI and MCIW, but not between the MCI and MCIW groups. Participants without anxiety were more likely to engage in physical activity than those with anxiety. This difference was significant, $t(3171)=5.04, p<0.001, r=0.09$. The same pattern was shown for symptoms of depression, as participants without depression were significantly more likely to engage in physical activity compared to those with depression, $t(3171)=7.72, p<0.001$, $r=0.14$.

\section{Service use}

Cognitive status was not related to service use, as the difference between groups was not significant, $F(2,3170)=9.80, p=0.838$. However, significant differences in service use were found between participants with anxiety and those without anxiety, $t(180.00)=-3.71, p<0.001, r=0.27$ (equal variances not assumed), where participants with anxiety had a higher service use than those who did not report anxiety. Participants with depression had a significantly higher service use score than participants who were not depressed, $t(1423.86)$ $=-2.62, p=0.009, r=0.06$ (equal variances not assumed).

\section{Instrumental activities of daily living}

A significant difference between participants categorized as NCI, MCI, and MCIW was found for IADL score, $F(2,369.83)=10.25, p<0.001$ (equal variances not assumed), with participants in the MCI category reporting the most difficulties, followed by the MCIW category and those in the NCI category reporting the least. Planned contrasts showed that participants with MCI and MCIW experienced more difficulties with IADLs than participants with NCI, and that this difference was significant, $t(507.78)=4.05$, $p<0.001, r=0.19$ (equal variances not assumed). The difference in IADL score between participants with MCI and MCIW was also significant, $t(394.81)=-3.11, p=0.002, r=0.15$ (equal variances not assumed). Games-Howell procedures confirmed that there were significant mean differences between participants in the NCI and MCI categories, and between participants categorized as MCI and MCIW, but there was no significant difference in IADL score between participants with NCI and those categorized as MCIW. There was a significant difference in IADL score between participants with anxiety and those, $t(175.10)=-7.84, p<0.001, r=0.51$ (equal variances not assumed). Similarly, participants with depression experienced significantly more IADL difficulties than those without depression, $t(1176.27)=-$ $10.88, p<0.001, r=0.30$ (equal variances not assumed).

\section{Health as a latent variable}

Structural equation modeling was used to assess health as a latent variable that combined the various health-related measures outlined above. The participants were randomly allocated into two groups to allow for a model calibration group and a model validation group. The hypothesized model included number of health conditions, physical activity, service use, IADL score and perceived health collapsed into two categories as observed variables that loaded on to a latent health variable. Preliminary sensitivity analyses suggested that the reliability for three IADL items (managing money, checking change, and following TV programs) was poor and consequently it was decided to remove these from the SEM analyses to increase the fit of the model. On calibration of the model, physical activity was found to reduce the fit of the model and this was subsequently removed. The final model included two IADL items (heavy housework, and shopping and carrying heavy bags), number of health conditions, service use, and perceived health, which loaded on to the latent health variable. The loadings of the observed variables can be seen in Table 3. The latent health variable was regressed upon cognitive status and mood, with mood acting as a mediating variable between health and cognitive status (Fig. 2). Anxiety and depression were tested separately for each of the three cognitive status categories, with each model being compared to a null model that specified no relationships between the variables. Model fitting statistics can be seen in Table 4. 
Table 3

Factor loadings of observed measures of health on to the latent health variable

\begin{tabular}{lccc}
\hline & Estimate & Standard error & $p$-value \\
\hline IADL1 & 0.63 & 0.026 & 0.000 \\
IADL2 & 0.62 & 0.025 & 0.000 \\
Total health conditions & 0.64 & 0.019 & 0.000 \\
Total service use & 0.26 & 0.009 & 0.000 \\
Perceived health & 0.57 & 0.020 & 0.000 \\
\hline
\end{tabular}

IADL, instrumental activities of daily living.

The results of the SEM analyses suggested that health had a direct effect on being categorized as having MCI $(b=0.07, p=0.021)$, but not on being categorized as MCIW $(b=-0.049, p=0.186)$ or NCI
Table 4

Model fitting statistics for each structural equation model

\begin{tabular}{llccccc}
\hline Model & & $\chi^{2}$ & $p$-value & $d f$ & RMSEA & CFI \\
\hline Anxiety & NCI & 26.75 & 0.008 & 12 & 0.028 & 0.989 \\
& MCI & 14.81 & 0.248 & 12 & 0.012 & 0.998 \\
& MCIW & 28.69 & 0.044 & 12 & 0.029 & 0.988 \\
Depression & NCI & 31.14 & 0.002 & 12 & 0.031 & 0.986 \\
& MCI & 19.17 & 0.085 & 12 & 0.019 & 0.995 \\
& MCIW & 34.24 & 0.001 & 12 & 0.035 & 0.986 \\
\hline
\end{tabular}

NCI, no cognitive impairment; MCI, objective cognitive impairment, intact general cognition, intact activities of daily living, subjective memory complaint reported by the participant, absence of dementia; MCIW, objective cognitive impairment, intact general cognition, intact activities of daily living, absence of subjective memory complaint, absence of dementia; RMSEA, root mean square error of approximation; CFI, comparative fit index.

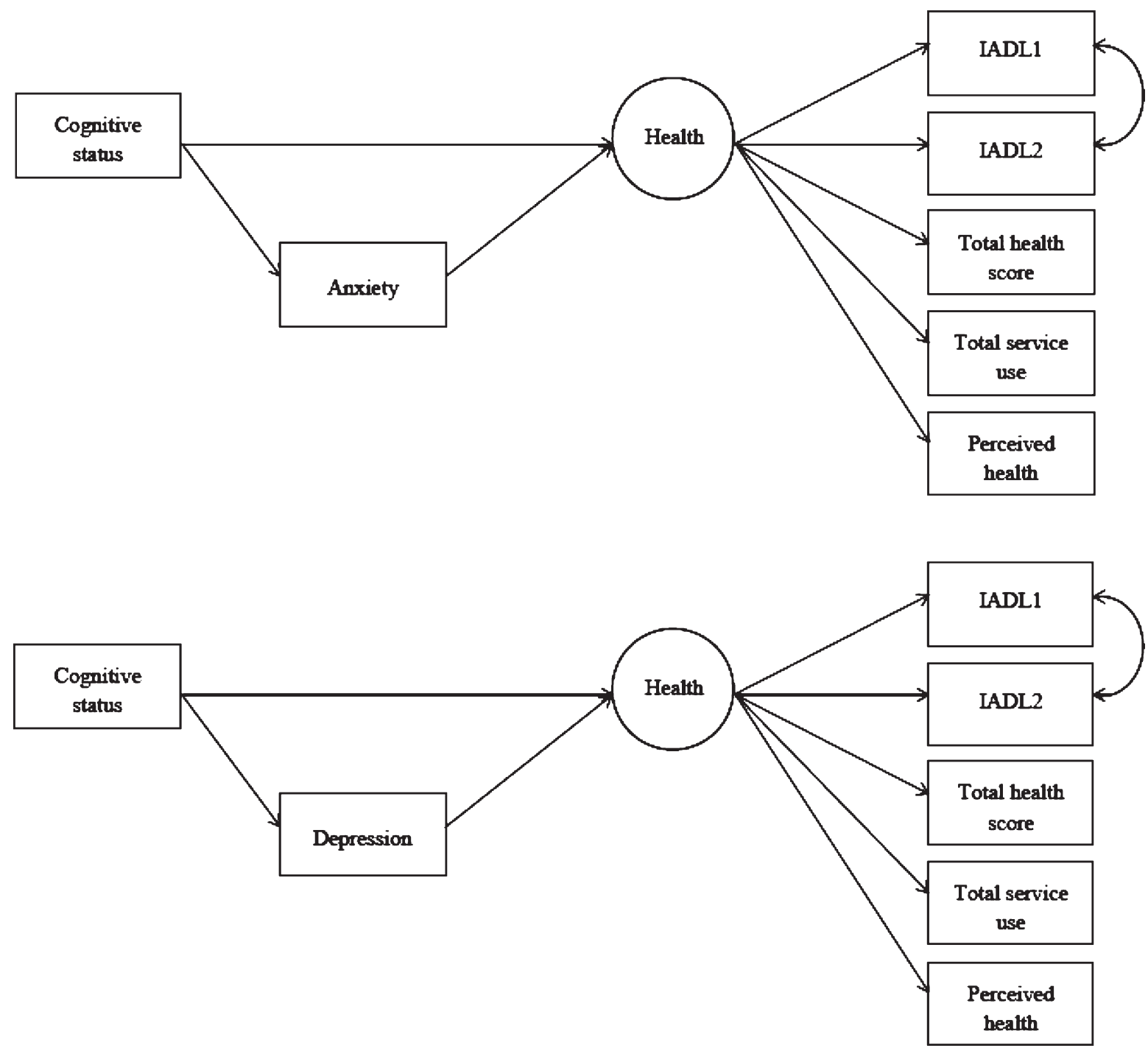

Fig. 2. Final structural equation model solution showing anxiety (top; non-significant) and depression (bottom; significant) as mediators. 
$(b=-0.01, p=0.893)$. Anxiety was not associated with being categorized as NCI $(b=-0.03, p=0.613)$, MCIW $(b=-0.01, p=0.914)$, or MCI $(b=0.04$, $p=0.372)$. Anxiety did not mediate the relationships between health and MCI ( $b=0.02, p=0.374)$, health and MCIW ( $b=0.00, p=0.914)$, or health and being NCI $(b=-0.01, p=0.614)$.

The risk of experiencing symptoms of depression was significantly decreased in people categorized as NCI $(b=-0.08, p=0.017)$, but was slightly increased in those classified as having $\mathrm{MCI}(b=0.09$, $p=0.003)$, and did not significantly change for people in the MCIW category $(b=-0.02, p=0.618)$. Depression significantly mediated the relationship between health and MCI $(b=0.30, p=0.005)$ and between health and NCI $(b=-0.03, p=0.021)$, but was not a mediator of the relationship between health and MCIW $(b=-0.01, p=0.618)$.

\section{DISCUSSION}

This study, one of the first to adopt a combination of proxy measures of health, has demonstrated that symptoms of depression mediate the relationship between mild cognitive impairment and health. Additionally, all measures of health were independently associated with mood, and perceived health, physical activity, and IADL difficulties were associated with cognitive status.

SEM showed that the latent variable of health was associated with being classified as having MCI, but not with the NCI or MCIW categories. Anxiety was not associated with the NCI, MCI, or MCIW categories, and did not mediate the relationship between health and cognitive status. However, the odds of experiencing symptoms of depression were decreased for people with NCI, were increased in those categorized as having MCI, and remained unchanged for participants in the MCIW category. The relationship between health and depression was only found in people with MCI and not in those in the MCIW category, suggesting that awareness of cognitive difficulties may be involved in this relationship. The findings regarding symptoms of anxiety remain unclear and further investigation with a larger sample is needed.

Our findings are concordant with previous literature, which has also shown an association between cognitive functioning and IADL difficulties [24], cognitive functioning and hospitalization [25], the association of overall medical burden and increased risk of depression [26], and that depression is associated with functional decline [27]. One clinical implication of these findings is that if mood problems are treated there are likely to be positive effects on how people perceive their own health, which in turn could improve functional status and reduce health and social service use [28]. Improvements to cognitive functioning could also improve self-perception of health; however, this would be more difficult to achieve.

Our findings concerning the participants in the MCIW group are particularly interesting. For this group of participants, there was no change in odds for reporting poor or fair perceived health, unlike participants in the MCI group. This suggests that perceived health and SMC may be part of a metacognitive process and that participants in this group appraise their health globally rather than focusing on their physical and cognitive health separately. Surprisingly, participants in the MCIW group reported fewer co-morbid health conditions than participants with MCI or without cognitive impairment. This finding could be due to these participants having fewer health problems, or alternatively this group of participants may be less aware of physical health problems, and consequently may not be accessing health services that could be beneficial to them.

This study has some limitations. First, the number of participants reporting anxiety was very small and this may have resulted in low statistical power. In addition, the numbers of participants in the cognitive status groups were unequal, which resulted in unequal variances across several statistical tests and may have contributed to the small effect size in some of the analyses. However, the statistical tests used are robust, which supports confidence in the findings. A further limitation is that the questions regarding anxiety in the CFAS Wales interview and the AGECAT algorithm [23] used to classify participants as having anxiety may not be sensitive enough to elicit the true nature of anxiety in older people. Anxiety in older people is difficult to measure [29] and relatively little is known about evidence-based approaches to measuring anxiety in this group [30], and so questions using a scaled response may have provided a better measure. However, participants classified as a sub-case of anxiety according to the AGECAT algorithm were considered to have anxiety in this study in order to capture milder and less frequent symptoms.

The measures of physical activity and health service use do not have a validated scoring system and 
instead, positive answers to questions asked in the CFAS Wales interview were summed to create composite scores. Validated measures of physical activity and health service use may have elicited clearer responses in order to create a more accurate picture of health in older people; however, at the time of development of the CFAS studies in the 1980s, these were unavailable, and the measures used are in line with other similar longitudinal studies. Additionally, due to the age of this measure, different opportunities for physical activity and changing tastes or interests over time mean that this measure may benefit from being refreshed to include activities that a modern cohort may endorse. For example, there was not a great deal of 'dancing' or 'heavy housework' reported.

The measure of health conditions was derived from the Charlson Co-Morbidity Index using the questions asked in the CFAS Wales interview. Recent research [31] has reported that the weightings for some health conditions should be adjusted to reflect the aging population, however, the reliability of our health measure using the original Charlson weightings [22] was found to be good and so the weightings were not adjusted in this study. Furthermore, the Charlson Comorbidity Index was developed for use with hospital administration data [32], but research suggests that it is applicable to self-report and questionnaire data [33] and the measure of health conditions used in these analyses may be treated confidently.

Despite these limitations, this study does have several significant strengths. The overall sample size is very large, included both community dwellers and those living in residential care, and had a response rate of approximately $50 \%$. In addition, extensive efforts were made to contact potential participants living in very rural and isolated areas, and people over 75 years of age were over-sampled to ensure that the oldest old were well represented.

Health is multi-dimensional, and using several aspects of health and health-related behaviors has added to the detail of the analysis. The use of welldefined cognitive categories is a further strength of this study. The MCI cognitive status group is based on criteria similar to those of Petersen [34], which will allow the results of this study to be compared to other studies. The MCIW cognitive status group allowed for investigation of the differences experienced by those who do not report SMC, by comparing participants in this group to those who meet traditional criteria for MCI that include a report of SMC, which has added to the emerging literature on subjective cognitive impairment.
Our findings show that health, mood problems, and cognitive functioning in older people are linked, and that interventions in one area may benefit other areas. For example, improvements in health may assist with preventing or reducing mood problems, which will also benefit cognition. In reducing mood problems, fewer burdens may be placed on health and social services, and this would benefit the wider community in freeing up health service resources. In addition, older people in receipt of health and social services are also likely to be cared for by family members $[35,36]$. Consequently, in helping to decrease the effects of anxiety and depression and need for services, the burden placed on family members could be reduced. Caregivers can develop avoidable health problems [37], which may be prevented through supporting those with symptoms of anxiety and depression.

This study has brought together several measures of health to provide a picture of the relationship between MCI, mood, and health in greater detail than has been seen in other research, allowing different conceptualizations of health to be investigated in relation to mood and cognition. The use of the MCI and MCIW categories has shown that perceptions of health extend to include cognitive functioning, which is an important finding given that physical and cognitive health are often treated very separately by patients and doctors alike. This is the first study to show that participants whose cognitive performance meets criteria for MCI but who do not report SMC also report fewer health problems than participants with MCI and those with normal cognitive functioning. Overall, this study has clarified the link between health and mood, which in turn influences cognitive functioning; with careful intervention and encouragement to maintain healthy lifestyles as people age it may be possible to ameliorate mood problems and reduce the risk of cognitive decline.

\section{ACKNOWLEDGMENTS}

The CFAS Wales study was funded by the Economic and Social Research Council (ESRC) (RES-060-25-0060) and HEFCW as 'Maintaining function and well-being in later life: a longitudinal cohort study'. The grant holders are Professors Woods (CI; Bangor), Clare (Exeter), Brayne, Matthews (Cambridge), Burholt, Phillips (Swansea), and Drs. Windle (Bangor), Bennett and McCracken (Liverpool). We are grateful to the NISCHR Clinical 
Research Centre for their assistance in tracing participants and in interviewing and in collecting blood samples, and to general practices in the study areas for their cooperation. We would like to thank the CFAS Wales population, their families and carers for their participation.

Authors' disclosures available online (http://j-alz. com/manuscript-disclosures/16-0611r2).

\section{REFERENCES}

[1] Matthews FE, Stephan BCM, McKeith IG, Bond J, Brayne C (2008) Two year progression from mild cognitive impairment to dementia: To what extent do different definitions agree? J Am Geriatr Soc 56, 1424-1433.

[2] Van der Linde R, Stephan BCM, Matthews FE, Brayne C, Savva GM (2010) Behavioural and psychological symptoms in the older population without dementia - relationship with socio-demographics, health and cognition. Biomed Central Geriatrics 10, 87.

[3] Lyketsos CG, Lopez O, Jones B, Fitzpatrick AL, Breitner J, DeKosky S (2002) Prevalence of neuropsychiatric symptoms in dementia and mild cognitive impairment: Results from the cardiovascular health study. JAMA 288, 14751483.

[4] Forsell Y, Palmer K, Fratiglioni L (2003) Psychiatric symptoms/syndromes in elderly persons with mild cognitive impairment. Data from a cross-sectional study. Acta Neurol Scand Suppl 179, 25-28.

[5] Yates JA, Clare L, Woods RT (2013) Mild cognitive impairment and mood: A systematic review. Rev Clin Gerontol 23, 317-356.

[6] Williamson GM, Schulz R (1992) Physical illness and symptoms of depression among elderly outpatients. Psychol Aging 7, 343-351.

[7] Williamson GM, Schulz R (1992) Pain, activity restriction and symptoms of depression among community-residing elderly adults. J Gerontol Psychosoc Sci 47, 367-372.

[8] Alexopoulos GS, Buckwalter K, Olin J, Martinez R, Wainscott C, Krishnan KR (2002) Comorbidity of late-life depression: An opportunity for research in mechanisms and treatment. Biol Psychiatry 52, 543-558.

[9] Simonsick EM, Wallace RB, Blazer DG, Berkman LF (1995) Depressive symptomology and hypertensionassociated morbidity and mortality in older adults. Psychosom Med 57, 427-435.

[10] May M, McCarron P, Stansfeld S, Ben-Shlomo Y, Gallacher J, Yarnell J, Davey Smith G, Elwood P, Ebrahim S (2002) Does psychological distress predict the risk of ischemic stroke and transient ischemic attack? The Caerphilly Study. Stroke 33, 7-12.

[11] Elwood PC, Pickering J, Bayer A, Gallacher JEJ (2002) Vascular disease and cognitive function in older men in the Caerphilly cohort. Age Ageing 31, 43-48.

[12] Ebly EM, Schaefer JP, Campbell NRC, Hogan DB (1998) Folate status, vascular disease and cognition in elderly Canadians. Age Ageing 27, 485-491.

[13] Morris MA, Jacques PF, Rosenberg IH, Selhub J (2007) Folate and vitamin B-12 status in relation to anemia, macrosytosis and cognitive impairment in older Americans in the age of folic acid fortification. Am J Clin Nutr $\mathbf{8 5}$, 193-200.
[14] Stephan BCM, Brayne C, Savva GM, Matthews FE, Medical Research Council Cognitive Function and Ageing Study (2011) Occurrence of medical co-morbidity in mild cognitive impairment: Implications for generalisation of MCI research. Age Ageing 40, 501-507.

[15] Laurin D, Verreault R, Lindsay J, MacPherson K, Rockwood K (2001) Physical activity and risk of cognitive impairment and dementia in elderly persons. Arch Neurol 58, 498-504.

[16] Stoddart H, Whitley E, Harvey I, Sharp D (2002) What determines the use of home care services by elderly people? Health Soc Care Community 10, 348-360.

[17] Yates JA, Clare L, Woods B (2015) Subjective memory complaints, mood and MCI: A follow-up study. Aging Ment Health, doi: 10.1080/13607863.2015.1081150

[18] Yates JA, Clare L, Woods B (2015) Subjective memory complaints are involved in the relationship between mood and mild cognitive impairment. J Alzheimers Dis $\mathbf{4 8}$, S115S123.

[19] Lenehan ME, Klekociuk SZ, Summers MJ (2012) Absence of a relationship between subjective memory complaint and objective memory impairment in mild cognitive impairment (MCI): Is it time to abandon subjective memory complaint as an MCI diagnostic criterion? Int Psychogeriatr 24, 1-10.

[20] Stephan BCM, Brayne C, McKeith IG, Bond J, Matthews FE (2008) Mild cognitive impairment in the older population: Who is missed and does it matter? Int J Geriatr Psychiatry 23, 863-871.

[21] Huppert FA, Brayne C, Gill C, Paykel ES, Beardsall L (1995) CAMCOG - a concise neuropsychological test to assist dementia diagnosis: Socio-demographic determinants in an elderly population sample. Br J Clin Psychol 34, 529541.

[22] Charlson ME, Pompei P, Ales KL, MacKenzie CR (1987) A new method of classifying prognostic comorbidity in longitudinal studies: Development and validation. J Chronic Dis 40, 373-383.

[23] Copeland JRM, Dewey ME, Griffiths-Jones HM (1986) A computerized psychiatric diagnostic system and case nomenclature for elderly subjects: GMS and AGECAT. Psychol Med 16, 89-99.

[24] Perneczky R, Pohl C, Sorg C, Hatmann J, Tosic N, Grimmer T, Heitele S, Kurz A (2006) Impairment of activities of daily living requiring memory or complex reasoning as part of the MCI syndrome. Int J Geriatr Psychiatry 21, 158-162.

[25] Weiler PG, Lubben JE, Chi I (1991) Cognitive impairment and hospital use. Am J Public Health 81, 1153-1157.

[26] Alexopoulos GS (2005) Depression in the elderly. Lancet 365, 1961-1970.

[27] Stuck AE, Walthert JM, Nikolaus T, Bula CJ, Hohmann C, Beck JC (1999) Risk factors for functional status decline in community-living elderly people: A systematic literature review. Soc Sci Med 48, 445-469.

[28] Bond J, Dickinson HO, Matthews F, Jagger C, Brayne C (2006) Self-rated health as a predictor of death, functional and cognitive impairment: A longitudinal cohort study. Eur J Ageing 3, 193-206.

[29] Lenze EJ, Wetherell JL (2009) Bringing the bedside to the bench, and then to the community: A prospectus for intervention research in late-life anxiety disorders. Int J Geriatr Psychiatry 24, 1-14.

[30] Therrien Z, Hunsley J (2012) Assessment of anxiety in older adults: A systematic review of commonly used measures. Aging Ment Health 16, 1-16.

[31] Quan H, Li B, Couris CM, Fushimi K, Graham P, Hider P, Januel J, Sundararajan V (2011) Updating and validating the 
Charlson Comorbidity Index and score for risk adjustment in hospital discharge abstracts using data from six countries. Am J Epidemiol 173, 676-682.

[32] Chaudhry S, Jin L, Meltzer D (2005) Use of a self-reportgenerated Charlson Comorbidity Index for predicting mortality. Med Care 43, 607-615.

[33] Katz JN, Chang LC, Sangha O, Fossel AH, Bates DW (1996) Can comorbidity be measured by questionnaire rather than medical record review? Med Care 34, 73-84.
[34] Petersen RC (2004) Mild cognitive impairment as a diagnostic entity. J Intern Med 256, 183-194.

[35] Luker KA, Perkins ES (1987) The elderly at home: Service needs and provision. J R Coll Gen Pract 37, 248-250.

[36] Wenger GC (1985) Care in the community. Changes in dependency and use of domiciliary service: A longitudinal perspective. Aging Soc 5, 143-159.

[37] Simon C (2001) Informal carers and the primary care team. Br J Gen Pract 51, 920-923. 